\title{
Performance of a Ka-Band Transponder Breadboard for Deep-Space Applications
}

\author{
N. R. Mysoor, J. P. Lane, S. Kayalar, and A. W. Kermode \\ Spacecraft Telecommunications Equipment Section
}

\begin{abstract}
This article summarizes the design concepts applied in the development of an advanced $\mathrm{Ka}$-band $(34.4 \mathrm{GHz} / 32 \mathrm{GHz})$ transponder breadboard for the next generation of space communications systems applications. The selected architecture upgrades the $X$-band $(7.2 \mathrm{GHz} / 8.4 \mathrm{GHz})$ deep-space transponder (DST) to provide $\mathrm{Ka}$-band up/Ka- and $\mathrm{X}$-band down capability. In addition, it can also be configured to provide $\mathrm{X}$-band up/Ka- and X-band down capability. The Ka-band transponder breadboard incorporates several state-of-the-art components, including sampling mixers, a Ka-band dielectric resonator oscillator, and microwave monolithic integrated circuits (MMICs). The MMICs that were tested in the breadboard include upconverters, downconverters, automatic gain control circuits, mixers, phase modulators, and amplifiers. The measured receiver dynamic range, tracking range, acquisition rate, static phase error, and phase jitter characteristics of the Ka-band breadboard interfaced to the advanced engineering model X-band DST are in good agreement with the expected performance. The results show a receiver tracking threshold of $-149 \mathrm{dBm}$ with a dynamic range of $80 \mathrm{~dB}$ and a downlink phase jitter of 7-deg rms. The analytical results of phase noise and Allan standard deviation are in good agreement with the experimental results.
\end{abstract}

\section{Introduction}

Telecommunications transponders for deep-space spacecraft applications [1,2] provide uplink command, turnaround ranging, differential one-way ranging (DOR), downlink telemetry, and radiometric capabilities. The Ka-band transponder breadboard provides these capabilities at a Ka-band uplink [3] frequency of $34.415 \mathrm{GHz}$ and a downlink frequency of $31.977 \mathrm{GHz}$. Operation at Ka-band allows the use of smaller antennas; it provides both a higher transmission bandwidth to allow a 4- to 6-dB improvement over $\mathrm{X}$-band on downlink data rate capability and increased accuracy in DOR applications. Furthermore, Kaband has a unique advantage for missions such as Solar Probe, Mercury, and Venus missions as it suffers negligible signal-to-noise degradation due to signal scintillation in the solar plasma. The signal-to-noise degradation at X-band is estimated to be about 5 to $10 \mathrm{~dB}$ in solar flux environment. To enable flexibility in spacecraft design and modularity in spacecraft hardware, the design was chosen to provide the Kaband functionality by adding conversion circuitry to the existing X-band (7.2-GHz/8.4-GHz) deep-space transponder (DST) design [2], as shown in Fig. 1. This modularity allows either X- or Ka-band uplink and $\mathrm{X}$ - and/or Ka-band downlink operation. This article describes the Ka-band transponder design, analysis, and breadboard performance results. The Ka-band transponder block diagram, functional requirements, 


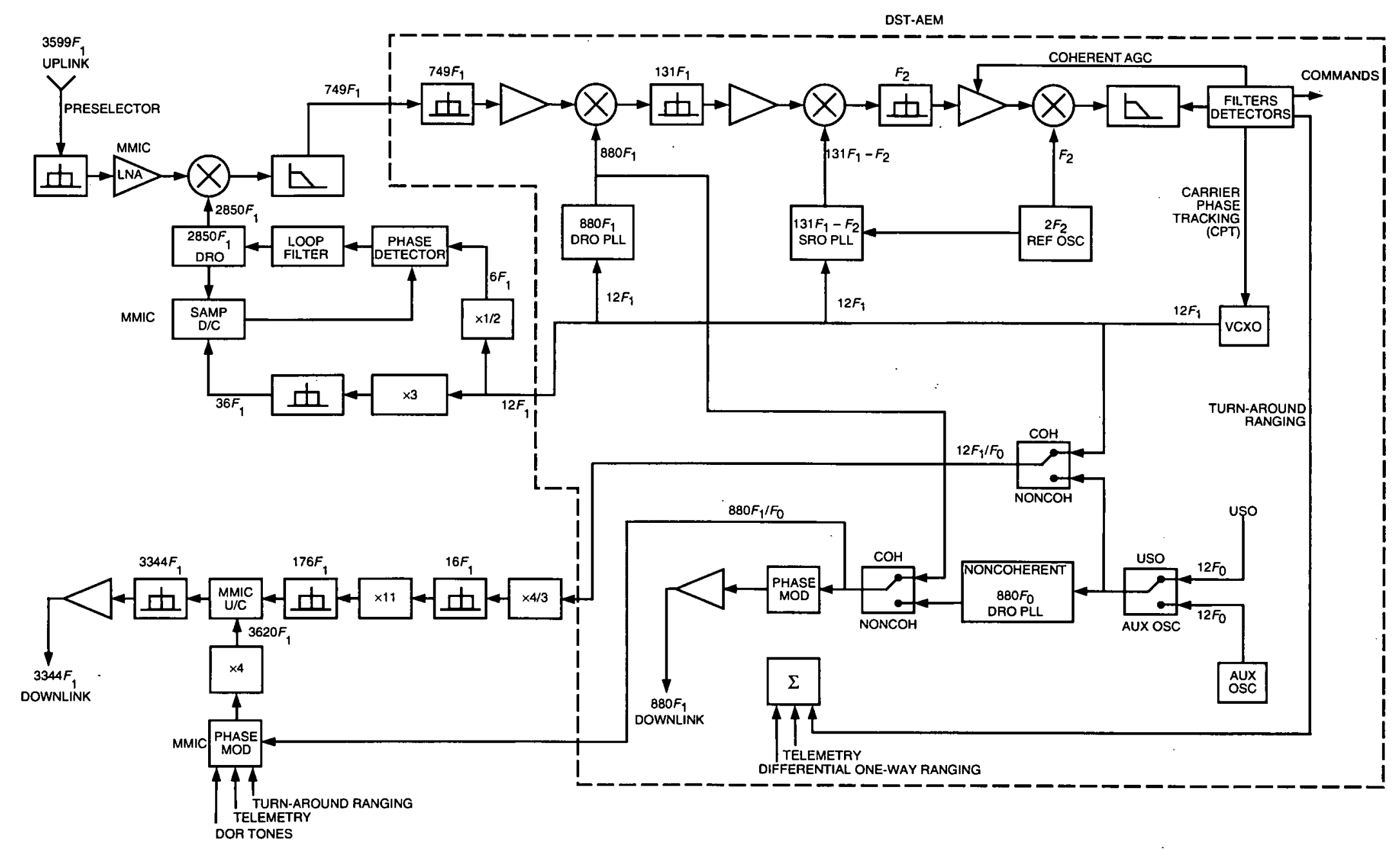

Fig. 1. Ka-band transponder functional block diagram. 
and design specifications are summarized in Section II. The transponder phase noise analysis is described in Section III. The breadboard performance results and conclusions are presented in Sections IV and V, respectively.

\section{Ka-Band Transponder Block Diagram and Functional Requirements}

\section{A. Block Diagram and Frequency Scheme}

The Ka-band transponder functional block diagram and frequency generation scheme are shown in Fig. 1. The Ka-band transponder design uses the X-band DST and Ka-band receiver and exciter circuitry to implement the transponder functions at Ka-band uplink and downlink frequencies $[2,3]$. The Ka-band receiver and exciter circuits are designed to interface directly with either the X-band DST breadboard or the X-band DST advanced engineering model (DST-AEM) developed by Motorola for JPL. The Ka-band receiver circuitry performs preselection and low-noise amplification at Ka-band and downconversion to the X-band DST uplink frequency. The Ka-band exciter circuitry performs telemetry, ranging, and DOR phase modulation on the X-band DST downlink reference and frequency multiplication and conversion to the Ka-band downlink frequency.

The design requirements for the Ka-band transponder and frequency translation ratios are listed in Table 1. The uplink and downlink frequencies are expressed in terms of the reference frequency, $F_{1}$. The exact $F_{1}$ frequency is determined by the Deep Space Network (DSN) channel in operation. The DSN channel frequencies $[2,3]$ selected for this implementation correspond to channel number 14 at $2 F_{1}$ with $F_{1}$ equal to $9.5625 \mathrm{MHz}$ for coherent mode, and channel number 16 at $2 F_{0}$ with $F_{0}$ equal to $9.565586 \mathrm{MHz}$ for noncoherent mode. The turnaround ratio of the uplink-to-downlink frequency is equal to $3599 / 3344(34.4 \mathrm{GHz} / 32 \mathrm{GHz})$. The criteria [3] used in the selection of the turnaround ratio include the maximum possible number of DSN channels for simultaneous X-and Ka-band operation, transmit/receive channel separation for diplexer implementation, central Ka-band allocation considerations, transponder implementation complexity, and uplink/downlink interference considerations.

1. X-Band Deep-Space Transponder Advanced Engineering Model Frequency Plan. The functional block diagram for the $\mathrm{X}$-band deep-space transponder advanced engineering model is shown in Fig. 1. The X-band receiver [2] is implemented as a double-conversion superheterodyne phase-lock carriertracking receiver with a fixed second intermediate frequency (IF). The first and second intermediate frequencies are at $131 F_{1}(1252.7 \mathrm{MHz})$ and $F_{2}(12.25 \mathrm{MHz})$, respectively. Coherent carrier automatic gain control (AGC) is employed in both of the IF sections to provide a constant signal plus noise at the carrier loop phase detector. The first local oscillator (LO) signal at $880 F_{1}(8415 \mathrm{MHz})$ and the second LO signal at $131 F_{1}-F_{2}$ are generated by a dielectric resonator oscillator (DRO) [4] and a surface acoustic wave resonator oscillator (SRO), respectively. Both of these oscillators are phase locked to the $12 F_{1}(114.75 \mathrm{MHz})$ voltage controlled oscillator (VCO). The $12 F_{1}$ VCO is in turn phase locked to the uplink carrier. The SRO phase-locked loop (PLL) consists of an SRO, a $\times 11$ multiplier, a $\div 6$ divider, two mixers, $\mathrm{a} \div 2$ divider, and a phase detector. The $\times 11$ multiplier and $\div 6$ divider are used in the SRO PLL to generate the frequencies $132 F_{1}$ and $2 F_{1}$ from $12_{1}$. The $2 F_{1}$ and the reference oscillator output at $2 F_{2}$ are applied to a mixer followed by a $\div 2$ divider to obtain a reference signal at $F_{1}+F_{2}$ to the SRO PLL phase detector. The SRO output at $131 F_{1}-F_{2}$ and the $132 F_{1}$ signals are applied to a mixer to obtain the second input signal at $F_{1}+F_{2}$ to the SRO PLL phase detector. A $\times 73$ multiplier and a $\div 3$ divider are used in the DRO PLL to generate reference signals at $836 F_{1}$ and $4 F_{1}$, respectively.

The coherent downlink carrier at $880 F_{1}$ is provided by the LO DRO when the DST is operating in the coherent mode from the VCO. In the noncoherent mode, an $880 F_{0}$ frequency is generated by the exciter DRO phase locked to the DST $12 F_{0}$ auxiliary oscillator (AUX OSC) or the external ultra-stable oscillator (USO). The noncoherent downlink signal is automatically selected by the receiver AGC function upon the absence of an uplink signal. An X-band phase modulator is used to phase modulate the downlink signal with telemetry signals, turnaround ranging, or DOR tones. 
Table 1. Ka-band transponder design requirements.

\begin{tabular}{|c|c|}
\hline Parameter & Design requirement \\
\hline \multicolumn{2}{|l|}{ Uplink frequency allocations } \\
\hline Ka-band uplink & $34,200-34,700 \mathrm{MHz}$, deep space \\
\hline X-band uplink & 7145-7190 MHz, deep space \\
\hline \multicolumn{2}{|l|}{ Downlink frequency allocations } \\
\hline Ka-band downlink & $31,800-32,300 \mathrm{MHz}$, deep space \\
\hline X-band downlink & 8400-8450 MHz, deep space \\
\hline \multicolumn{2}{|l|}{ Frequency translation ratios } \\
\hline Channel 14 Ka-band uplink frequency & $34,415.4375 \mathrm{MHz}\left(3599 F_{1}\right)$ \\
\hline Channel $14 \mathrm{X}$-band uplink frequency & $7162.3125 \mathrm{MHz}\left(749 F_{1}\right)$ \\
\hline X-band downlink & $880 / 3599(8415 \mathrm{MHz})$ \\
\hline Ka-band downlink & $3344 / 3599(31,977 \mathrm{MHz})$ \\
\hline \multicolumn{2}{|l|}{ Ka-band receiver parameters } \\
\hline Carrier threshold & $\leq-149 \mathrm{dBm}$, unmodulated carrier \\
\hline Dynamic range & $\geq 79 \mathrm{~dB}$ (carrier threshold to $-70 \mathrm{dBm}$ ) \\
\hline Noise figure at Ka-band receiver input & $\leq 6 \mathrm{~dB}$ \\
\hline Acquisition and tracking rate & $\geq 550 \mathrm{~Hz} / \mathrm{s}$ at carrier level $>-110 \mathrm{dBm}$ \\
\hline Tracking range & $\geq \pm 100 \mathrm{kHz}$ minimum \\
\hline Tracking error & $\leq 1 \mathrm{deg} / 40 \mathrm{kHz}$ at carrier level $>-110 \mathrm{dBm}$ \\
\hline Capture range & $\geq \pm 1.3 \mathrm{kHz}$ at carrier level $>-110 \mathrm{dBm}$ \\
\hline \multicolumn{2}{|l|}{ Ka-band exciter parameters } \\
\hline Frequency, coherent operation & $3344 / 3599 \times$ uplink frequency \\
\hline Frequency, noncoherent operation & $31,987.320988 \mathrm{MHz}\left(3344 F_{0}\right) \mid$ channel 16 \\
\hline RF output power level & $\geq+3 \mathrm{dBm}$ \\
\hline Output voltage standing wave ratio & $\leq 1.5: 1,50 \pm 5$ ohms \\
\hline Spurious signals & $\geq 60 \mathrm{dBc}$ below the carrier \\
\hline Modulation bandwidth & $\geq 50 \mathrm{MHz}$ at $\pm 0.5 \mathrm{~dB}$ \\
\hline Peak phase modulation index & $\pm 2.5 \mathrm{rad}$ at $\pm 8 \%$ linearity \\
\hline Modulation sensitivity & $2 \mathrm{rad}$ peak/volt peak \\
\hline Modulation index stability & $\pm 10 \%$ over $-20 \operatorname{deg} \mathrm{C}$ to $+75 \operatorname{deg} \mathrm{C}$ \\
\hline \multicolumn{2}{|l|}{ Modulation index } \\
\hline Turnaround ranging & 3-9 dB carrier suppression \\
\hline Telemetry & $0-15 \mathrm{~dB}$ carrier suppression \\
\hline DOR & $0-1.1 \mathrm{~dB}$ carrier suppression \\
\hline \multirow[t]{2}{*}{ Residual phase noise } & $\leq 8 \mathrm{deg} \mathrm{rms}$, coherent mode \\
\hline & $\leq 8 \mathrm{deg} \mathrm{rms}$, noncoherent mode \\
\hline Carrier phase delay variation & $\leq 12$ ns over $-20 \operatorname{deg} \mathrm{C}$ to $+75 \operatorname{deg} \mathrm{C}$ \\
\hline Differential phase delay variation & $\leq 2 \mathrm{~ns} \mathrm{Ka-} / \mathrm{X}$-band over $-20 \operatorname{deg} \mathrm{C}$ to $+75 \operatorname{deg} \mathrm{C}$ \\
\hline Ranging phase delay variation & $\leq 30$ ns over $-20 \mathrm{deg} \mathrm{C}$ to $+75 \mathrm{deg} \mathrm{C}$ \\
\hline
\end{tabular}

2. Ka-Band-to-X-Band Downconverter. The Ka-band receiver circuitry performs preselection and low-noise amplification at $3599 F_{1}(34.415 \mathrm{GHz})$ and downconversion to the $749 F_{1}(7.16 \mathrm{GHz})$ $\mathrm{X}$-band DST uplink frequency. A five-pole Chebychev waveguide iris preselector filter is used to filter the receiver Ka-band uplink frequency at $34.415 \mathrm{GHz}$, reject the Ka-band downlink frequency at $3344 F_{1}$ $(31.977 \mathrm{GHz})$, and attenuate the receiver LO leakage. After a waveguide-to-coax transition, a microwave 
monolithic integrated circuit (MMIC) low-noise amplifier (LNA) sets the noise figure of the receiver. A triple balanced hybrid mixer is used for downconversion to $\mathrm{X}$-band.

A phase-locked Ka-band DRO generates the LO signal at $2850 F_{1}(27.253 \mathrm{GHz})$ needed for the $3599 F_{1^{-}}$ to- $749 F_{1}$ downconversion. The phase-locked DRO yields lower spurious output and phase noise than could be achieved with a direct multiplication implementation. A hybrid MMIC sampling downconverter is used to sample the Ka-band LO output with a $36 F_{1}$ reference and downconvert it to $6 F_{1}$ for phase detection with a digital phase/frequency detector. The $6 F_{1}$ and $36 F_{1}$ loop references are derived from the $12 F_{1}$ reference with a divide-by-two analog Miller divider and a bipolar junction transistor (BJT) multiplier, respectively.

3. Ka-Band Exciter. The Ka-band exciter circuitry performs phase modulation on the $880 F_{1}$ $(8.415 \mathrm{GHz}) \mathrm{X}$-band DST downlink reference and frequency multiplication and conversion to the $3344 F_{1}$ $(31.977 \mathrm{GHz}) \mathrm{Ka}$-band downlink frequency. An X-band MMIC phase modulator [5] modulates the telemetry, ranging, and DOR signal on the X-Band DST downlink reference. The modulated X-band signal is multiplied to $3520 F_{1}(33.66 \mathrm{GHz})$ with a hybrid $\times 4$ varactor multiplier. The harmonics are filtered to reduce in-band spurious signals on the downlink. The $3520 F_{1}$ modulated signal is converted to the $3344 F_{1}$ downlink frequency by mixing it with a $176 F_{1}(1683 \mathrm{MHz})$ signal using a MMIC upconverter. The conversion spurious signals are filtered out. The $176 F_{1}$ reference for the conversion is generated from the $12 F_{1}$ reference signal from the DST with a Miller divider and step-recovery diode multiplier. The Miller divider multiplies the $12 F_{1}$ reference by $4 / 3$ to give $16 F_{1}$. The signal is multiplied with a $\times 11$ step-recovery diode circuit and filtered to produce the $176 F_{1}$ reference.

\section{B. Key Design Requirements}

The key design requirements for the Ka-band transponder are summarized in Table 1. These requirements apply to Ka-band receiver and exciter breadboards operating with the X-band DST-AEM. These requirements can be satisfied with no modifications to the X-band DST-AEM. The Ka-band receiver performance includes a noise figure of $5.4 \mathrm{~dB}$, an unmodulated carrier-tracking threshold of $-149 \mathrm{dBm}$, and a tracking range of $\pm 100 \mathrm{kHz}$ at the assigned channel frequency. The acquisition and tracking rate is specified to be at least $550 \mathrm{~Hz} / \mathrm{s}$. The specified nominal output power of the Ka-band exciter is $+3 \mathrm{dBm}$. The exciter output is phase modulated to a maximum phase deviation of $\pm 2.5 \mathrm{rad}$ with a radio frequency \pm 0.5 -dB modulation bandwidth of $50 \mathrm{MHz}$. The phase noise measured from $5 \mathrm{~Hz}$ to $25 \mathrm{MHz}$ is required to be less than 8-deg root mean square (rms) in both the coherent and the noncoherent modes.

\section{Ka-Band Transponder Phase Noise Analysis and Results}

The downlink phase-noise power spectral density of the Ka-band transponder is predicted using noise models [6-11] for various devices and the loop transfer functions in a custom JPL software package. A complete description of the phase noise model is given in Appendix A, and carrier threshold calculations are given in Appendix B. The phase noise models for the Ka-band receiver and exciter are shown in Fig. A-1. The phase noise outputs of the local oscillator loops are used in the receiver loop and the exciter to determine the resultant downlink phase noise. The loop transfer functions modify the noise spectral density of the individual contributors when referenced to the loop output. The models for phase noise contributors common to all loops are developed first. These contributors include oscillators, varactors, phase detectors, buffer amplifiers, multipliers, dividers, operational amplifiers, power converter circuits, regulators, and phase modulators. These devices are modeled with the appropriate white phase, flicker phase, white frequency, and flicker frequency noise characteristics. Each contributor is shown in the receiver and exciter block diagram (Fig. A-1) as an ideal component plus an additive block that represents the phase-noise power spectral density of the component. As shown in the block diagram, the phase noise density of each contributor is modified by the transfer function from the contributor to the output. For example, the noise at the phase detector input will be modified by the receiver loop transfer function, which is a low-pass response, whereas the noise at the $12 F_{1}$ VCO output will be modified by the loop error 
transfer function, which is a high-pass response. The receiver phase noise at the phase-locked VCO output is predicted by first calculating the contributions from the Ka-band, X-band, and L-band (1240.44 MHz) local oscillator circuits and using these phase noise density functions in the receiver loop. The receiver VCO output phase noise density thus obtained is used in the exciter to predict the coherent downlink phase noise density at Ka-band. The-Ka-band downlink phase noise densities for the noncoherent AUX OSC mode are obtained by replacing the receiver VCO phase noise density by the AUX OSC oscillator phase noise density. The predicted theoretical phase noise results are compared to the measured results in the following section.

\section{Experimental Results}

The Ka-band transponder breadboard (Fig. 1), consisting of the Ka-band-to-X-band downconverter, Ka-band exciter, and DST-AEM, was implemented, and performance characterization was accomplished. The evaluation measurements include receiver tracking-threshold sensitivity, static phase errors for Ka-band uplink frequency offset, swept acquisition characteristics, and AGC versus uplink signal level. All measurements were made at a room temperature of $25 \mathrm{deg} \mathrm{C}$. The measured tracking-threshold sensitivity at the receiver best-lock frequency, approximately at channel center, is $-149 \mathrm{dBm}$. The measured receiver threshold characteristics show good correlation with expected performance (Appendix B) over the tracking range, as shown in Fig. 2. The receiver acquisition characteristics were measured at an input signal level of $-110 \mathrm{dBm}$. The measured values for tracking range and tracking rate are $\pm 1.2 \mathrm{MHz}$ at design center frequency and $800 \mathrm{~Hz} / \mathrm{s}$, respectively, and they meet the specified requirements (Table 1). Figure 3 shows a linear relationship for the static phase error voltage versus uplink frequency offset over the receiver tracking range. The AGC loop-filter amplifier output voltage controls the gain in the first and second IF amplifiers. The AGC voltage versus uplink signal level at the best-lock frequency and at frequency offsets of $\pm 1.2 \mathrm{MHz}$ from best-lock frequency are shown in Fig. 4 . As the receiver input signal varies from a strong signal level $(-70 \mathrm{dBm})$ to the threshold level, the AGC control voltage varies approximately linearly with better than 5 percent linearity. No receiver false lock or self-lock resulted during the test phase.

In the coherent carrier mode, residual phase noise is defined for a noise-free received-signal case. The phase noise on the downlink carrier signal consists primarily of contributions from the four phase-locked oscillators, $12 F_{1}$ VCO, SRO, X-band DRO, and Ka-band DRO, used in the Ka-band transponder implementation. Individual phase-noise power spectral-density functions for these contributors are used in a comprehensive computer program (Appendix A) to predict the phase noise of the closed-loop receiver. Total residual phase noise in the output is the mean square sum of all noise sources. The predicted phase noise for the Ka-band transponder in the coherent mode is compared to the measured results in Fig. 5.

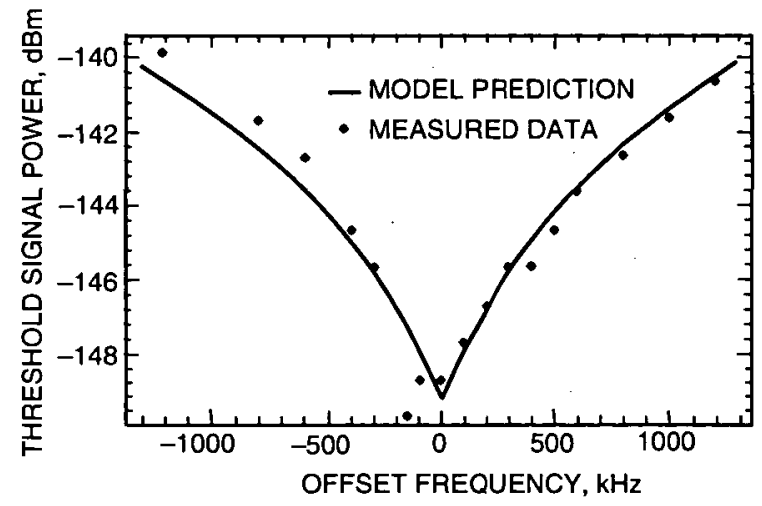

Fig. 2. Ka-band transponder carrier tracking threshold versus offset frequency.

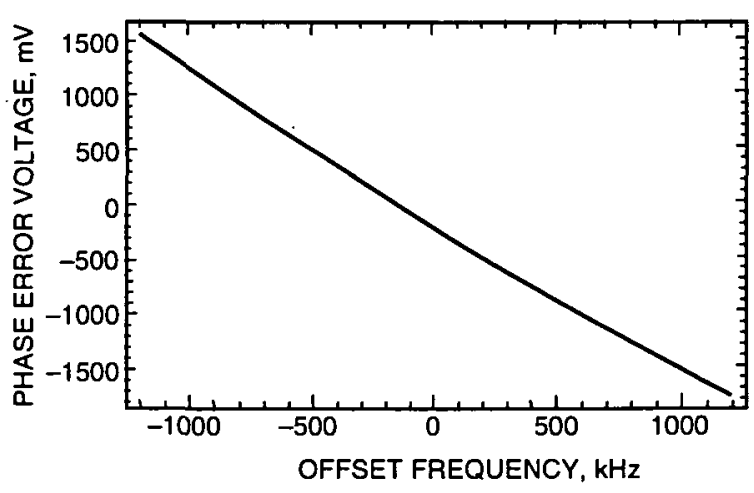

Fig. 3. Ka-band static phase error voltage versus offset frequency. 


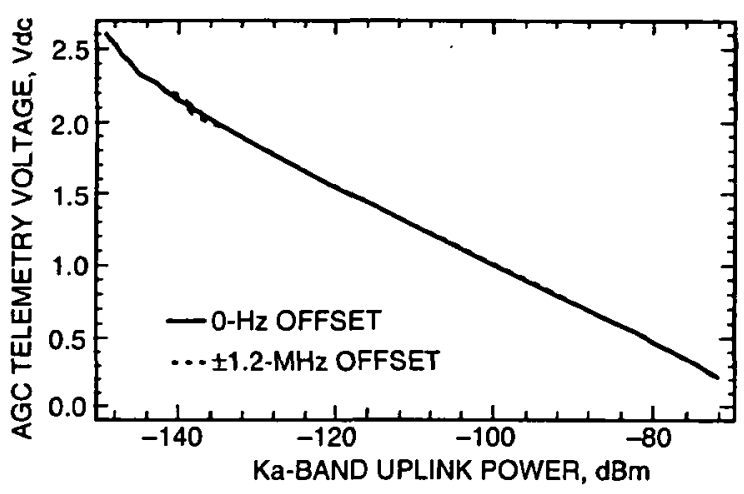

Fig. 4. Ka-band transponder AGC voltage versus uplink signal level.

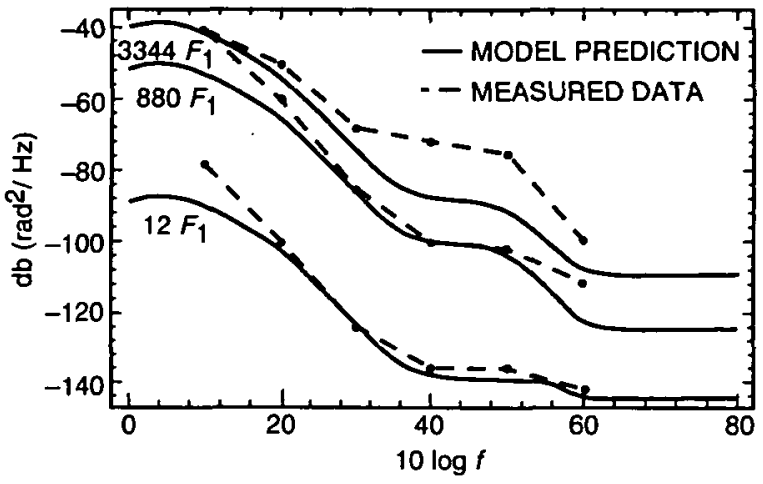

Fig. 5. Comparison of theoretical and measured coherent-mode phase noise densities (uplink = $-100 \mathrm{dBm}$ ).

Table 2. Predicted phase jitter and Allan deviation for coherent and noncoherent modes.

\begin{tabular}{|c|c|c|c|c|c|}
\hline \multirow{2}{*}{ Mode } & \multirow{2}{*}{ Frequency } & \multirow{2}{*}{$\begin{array}{c}\text { Phase jitter } \\
(5 \mathrm{~Hz}-25 \mathrm{MHz}), \\
\text { deg rms }\end{array}$} & \multicolumn{3}{|c|}{ Allan deviation } \\
\hline & & & $T=0.01 \mathrm{~s}$ & $T=1 \mathrm{~s}$ & $T=1000 \mathrm{~s}$ \\
\hline Coherent & $12 F_{1}$ & 0.02 & $1.5 \times 10^{-10}$ & $1.3 \times 10^{-12}$ & $9.3 \times 10^{-16}$ \\
\hline \multirow[t]{2}{*}{$(P=-100 \mathrm{dBm})$} & $880 F_{1}$ & 0.80 & $3.6 \times 10^{-11}$ & $4.6 \times 10^{-13}$ & $4.5 \times 10^{-16}$ \\
\hline & $3344 F_{1}$ & 3.10 & $4.6 \times 10^{-11}$ & $5.3 \times 10^{-13}$ & $4.9 \times 10^{-16}$ \\
\hline Noncoherent & $12 F_{1}$ & 0.08 & $6.1 \times 10^{-10}$ & $3.2 \times 10^{-11}$ & $3.2 \times 10^{-11}$ \\
\hline \multirow[t]{2}{*}{ (AUX OSC) } & $880 F_{1}$ & 1.98 & $4.8 \times 10^{-11}$ & $3.2 \times 10^{-11}$ & $3.2 \times 10^{-11}$ \\
\hline & $3344 F_{1}$ & 7.60 & $5.8 \times 10^{-11}$ & $3.2 \times 10^{-11}$ & $3.2 \times 10^{-11}$ \\
\hline
\end{tabular}

In the intervals between $5 \mathrm{~Hz}$ and $25 \mathrm{MHz}$ on each side of the carrier, the rms phase noise is $7 \mathrm{deg}$, which is below the maximum allowable 8-deg rms for coherent downlink. The dominant contributor to this rms phase noise is the $12 F_{1}$ VCO and the $\times 4$ multiplication process from X-band to Ka-band; the remaining contributions are less than 10 percent of the VCO contribution. Predicted rms phase noise and Allan deviation are presented in Table 2. The results of the analysis indicate that the coherent mode specifications will be met for both the rms phase noise and Allan deviation. The receiver PLL band limits the VCO spectrum, thus providing the superior performance in the coherent mode.

A comparison of measured-to-calculated Allan standard deviation characteristics as a function of integration time is shown in Fig. 6. The measured Allan standard-deviation values for the Ka-band transponder breadboard are in good agreement with the predicted values. Figure 7 shows good agreement between the theoretical and measured phase-noise density curves for the noncoherent AUX OSC mode operation.

A GaAs MMIC phase modulator developed under a Small Business Innovative Research Program contract at Pacific Monolithics, Inc., was used to modulate the X-band reference signal and upconvert ( $\times 4$ multiplication) it to a Ka-band downlink signal, as shown in Fig. 1. The modulator MMIC chip incorporates a single-stage input buffer amplifier and a three-stage lumped-element hybrid-coupled reflection phase shifter with metal-gate Schottky field-effect transistor (MESFET) varactors to provide a phase deviation of $\pm 2.5 \mathrm{rad}$ with better than 3-percent linearity. The chip size is $2.49 \times 0.91 \mathrm{~mm}$. Sinusoidal and square modulating waveforms were applied to the phase modulator, and their resulting spectra $[12,13]$ were monitored on a calibrated spectrum analyzer. All measurements were performed at $25 \mathrm{deg} C$ 


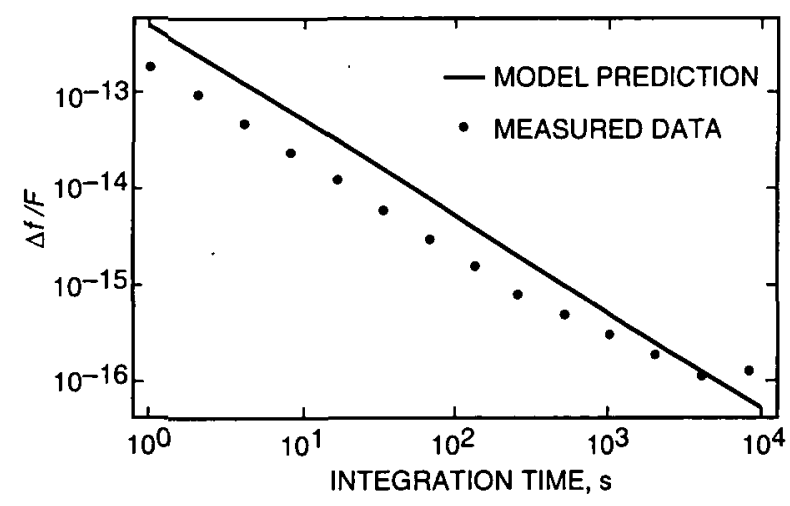

Fig. 6. Ka-band transponder Allan deviation versus integration time.

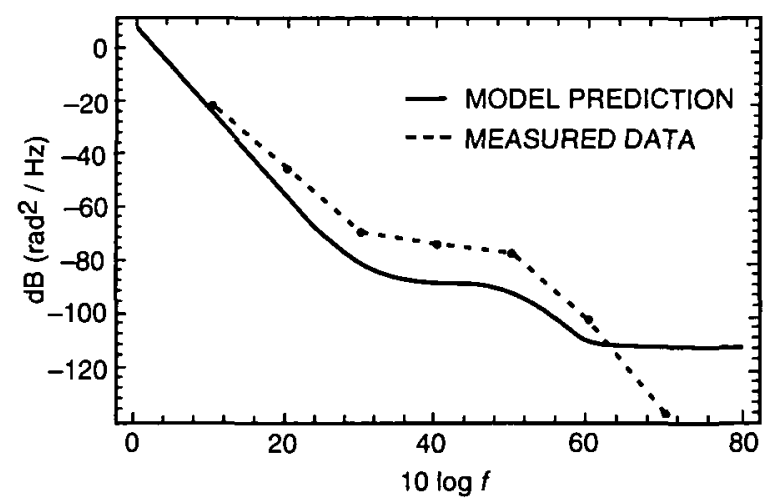

Fig. 7. Comparison of theoretical and measured noncoherent-mode phase noise densities.

and with a carrier frequency of $31,977 \mathrm{MHz}$. A comparison of measured and predicted carrier, first, second, third, and fourth side-band levels for the case of sinusoidal modulation is shown in Fig. 8. A modulation frequency of $100 \mathrm{kHz}$ was used in the measurements. The peak phase modulation index, $\beta$, ranges from 0.2 to $2.4 \mathrm{rad}$. The predicted relative carrier and side-band levels were computed $[12,14]$ by evaluating the appropriate Bessel functions of the first kind of order $n, J_{n}(\beta)$. The subscript $n$ is an integer. It represents the carrier for $n=0$ and the side bands for $n \neq 0$. The relative carrier level for a given modulation index is determined by evaluating $20 \log \left|J_{0}(\beta)\right|$. Similarly, the relative side-band levels are determined by evaluating $20 \log \left|J_{n}(\beta)\right|$ for $|n|>0$. Figure 8 shows excellent agreement between theory and measurement for sinusoidal phase modulating waves. Negligible amplitude modulation distortion was observed in this case.

A comparison of measured and predicted carrier, first, third, and fifth side-band levels for the case of square-wave modulation is shown in Fig. 9. A modulation frequency of $100 \mathrm{kHz}$ was used in the measurements. The relative carrier level in decibels is determined by evaluating $20 \log |\cos (\beta)|$, where $\beta$ is the peak modulation index. The relative side-band levels is determined by evaluating $20 \log |2 \sin (\beta) /(n \pi)|$, where $n=1,3,5, \cdots$, is the number of the side band. Good agreement between predicted and measured square-wave modulation results is shown in Fig. 9.

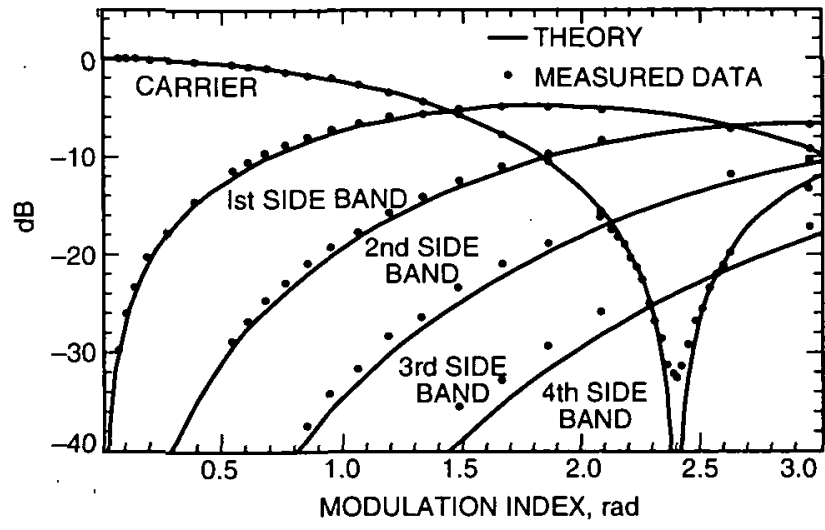

Fig. 8. Measured and predicted Ka-band carrier, first, second, third, and fourth side band levels versus phase modulation index for the case of a sinusoidal modulating wave of $100-\mathrm{kHz}$ frequency.

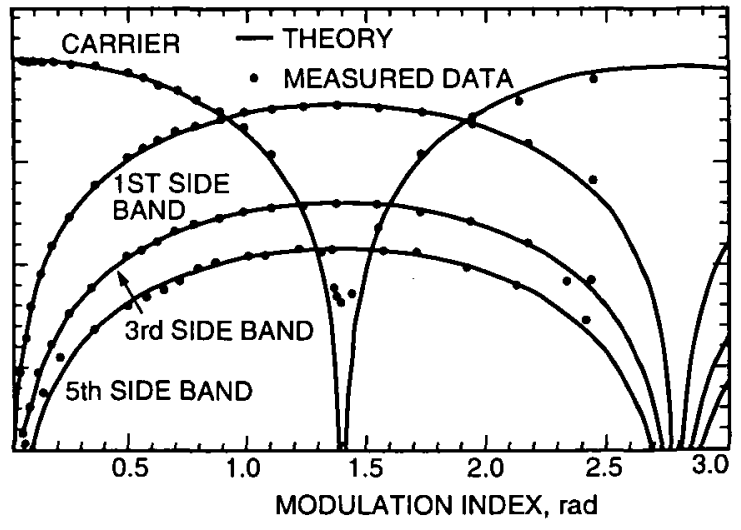

Fig. 9. Measured and predicted Ka-band carrier, first, third, and fifth side band levels versus peak phase modulation index for the case of a square modulating wave of $100-\mathrm{kHz}$ frequency. 


\section{Conclusions}

Design concepts and system architecture for a high-performance Ka-band transponder for deep-space spacecraft applications have been presented. The Ka-band transponder has been successfully breadboarded and evaluated. New technologies, such as a Ka-band DRO, X-band MMIC phase modulator, MMIC amplifiers, MMIC upconverter, and sampling mixers, have been integrated into the design. The Telecommunication Development Laboratory measurements on the breadboard transponder achieved a threshold level of $-149 \mathrm{dBm}$ with a dynamic range of $80 \mathrm{~dB}$ and excellent acquisition and tracking characteristics. The measured phase noise, Allan standard deviation, and phase jitter data are in good agreement with the predicted characteristics. Measured carrier and relative side-band amplitudes resulting from phase modulation of the Ka-band downlink signal by sinusoidal and square-wave modulating functions agree well with the predicted results with negligible amplitude modulation distortion.

\section{References}

[1] J. H. Yuen, Deep Space Telecommunications Systems Engineering, New York: Plenum Press, 1983.

[2] N. R. Mysoor, J. D. Perret, and A. W. Kermode, "Design Concepts and Performance of NASA X-Band (7162 MHz/8415 MHz) Transponder for DeepSpace Spacecraft Applications," The Telecommunications and Data Acquisition Progress Report 42-104, October-December 1990, Jet Propulsion Laboratory, Pasadena, California, pp. 247-256, February 15, 1991.

[3] J. A. Koukos, "Selection of Ka-Band Transponder Turnaround Frequency Ratios," Report of the Proceedings of RF and Modulation Subpanel $1 E$ Meeting at the German Space Operations Center, September 20-24, 1993, CCSDS B20.0-Y-1, Consultative Committee for Space Data Systems, Oberpfaffenhofen, Germany, February 1994.

[4] N. R. Mysoor, "An Electronically Tuned, Stable $8415 \mathrm{MHz}$ Dielectric Resonator FET Oscillator for Space Applications," Proc. IEEE 1990 Aerospace Applications Conference, Vail, Colorado, February 5-9, 1990.

[5] N. R. Mysoor and F. Ali, "Miniature X-Band GaAs MMIC Analog and Digital Modulators for Spaceborne Communications Applications," Technology 2001 Conference Proceedings, NASA Conference Publication 3136, vol. 1, San Jose, California, pp. 82-88, December 3-5, 1991.

[6] F. M. Gardner, Phaselock Techniques, New York: John Wiley, 1979.

17) A. Blanchard, Phase-Locked-Loops: Applications to Coherent Receiver Design, New York: John Wiley and Sons, 1976.

[8] J. P. Frazier and J. Page, "Phase-Lock Loop Frequency Acquisition Study," IRE Trans., vol. 8, pp. 210-227, September 1962.

[9] D. B. Leeson, "A Simple Model of Feedback Oscillator Noise Spectrum," Proc. IEEE, vol. 54, pp. 329-330, February 1966.

[10] J. A. Barnes, A. R. Chi, and L. S. Cutler, Characterization of Frequency Stability, National Bureau of Standards Technical Note 394, Washington, DC: National Bureau of Standards, October 1970. 
[11] D. W. Allan, "Time and Frequency (Time-Domain) Characterization, Estimation, and Prediction of Precision Clocks and Oscillators," IEEE Transactions on Ultrasonics, Ferroelectronics, and Frequency Control, vol. UFFC-34, no. 6, pp. 647-654, November 1987.

[12] N. R. Mysoor and R. O. Mueller, "Performance of a 300-Degree Linear Ana$\log$ Phase Modulator for Communications Applications," Proc. of IEEE 1993 Aerospace Applications Conference, Steamboat Springs, Colorado, pp. 43-52, February 1-5, 1993.

[13] E. A. Whitman, "Phase Modulation Measurement Techniques for Improved Accuracy," Microwave Journal, vol. 6, pp. 113-116, June 1978.

[14] F. Stocklin, Relative Side-Band Amplitudes vs. Modulation Index for Common Functions Using Frequency and Phase Modulation, Goddard Space Flight Center, distributed by National Technical Information Service, U.S. Department of Commerce, Springfield, Virginia, November 1973.

\section{Appendix A \\ Phase Noise Models}

The phase noise models [6-11] and the equations used to calculate the downlink residual phase noise of the Ka-band transponder in the coherent and noncoherent modes are presented in this appendix.

A block diagram of the Ka-band receiver and exciter phase noise models along with the major phase noise contributors are shown in Fig. A-1. Each contributor is shown in the block diagram as an ideal component plus an additive block that represents the phase-noise power spectral density of the component.

\section{Phase Noise Models of Individual Contributors}

A brief description of the phase noise models for the contributing circuits to all common loops is given below.

(1) Buffer Amplifiers/Multipliers/Dividers/Phase Detector: The buffer amplifiers are modeled to have a $1 / f$ Halford noise plus a thermal noise floor. The thermal noise floor is set by the input power level and the noise figure of the amplifier. The phase noise density of operational amplifiers used in the loop filters is modeled similarly. Also, phase noise contributions of multipliers, dividers, and phase detectors are obtained from a similar model.

(2) Oscillators: The oscillator phase noise density is based on Leeson's model [9]. The phase noise model has a $1 / f^{3}$ slope from a $1-\mathrm{Hz}$ offset from the oscillator center frequency, followed by a $1 / f$ slope and a thermal noise floor. The model uses the flicker $1 / f$ noise corner frequency of the FET, the noise figure of the FET, the bandwidth of the oscillator, and the varactor noise. 


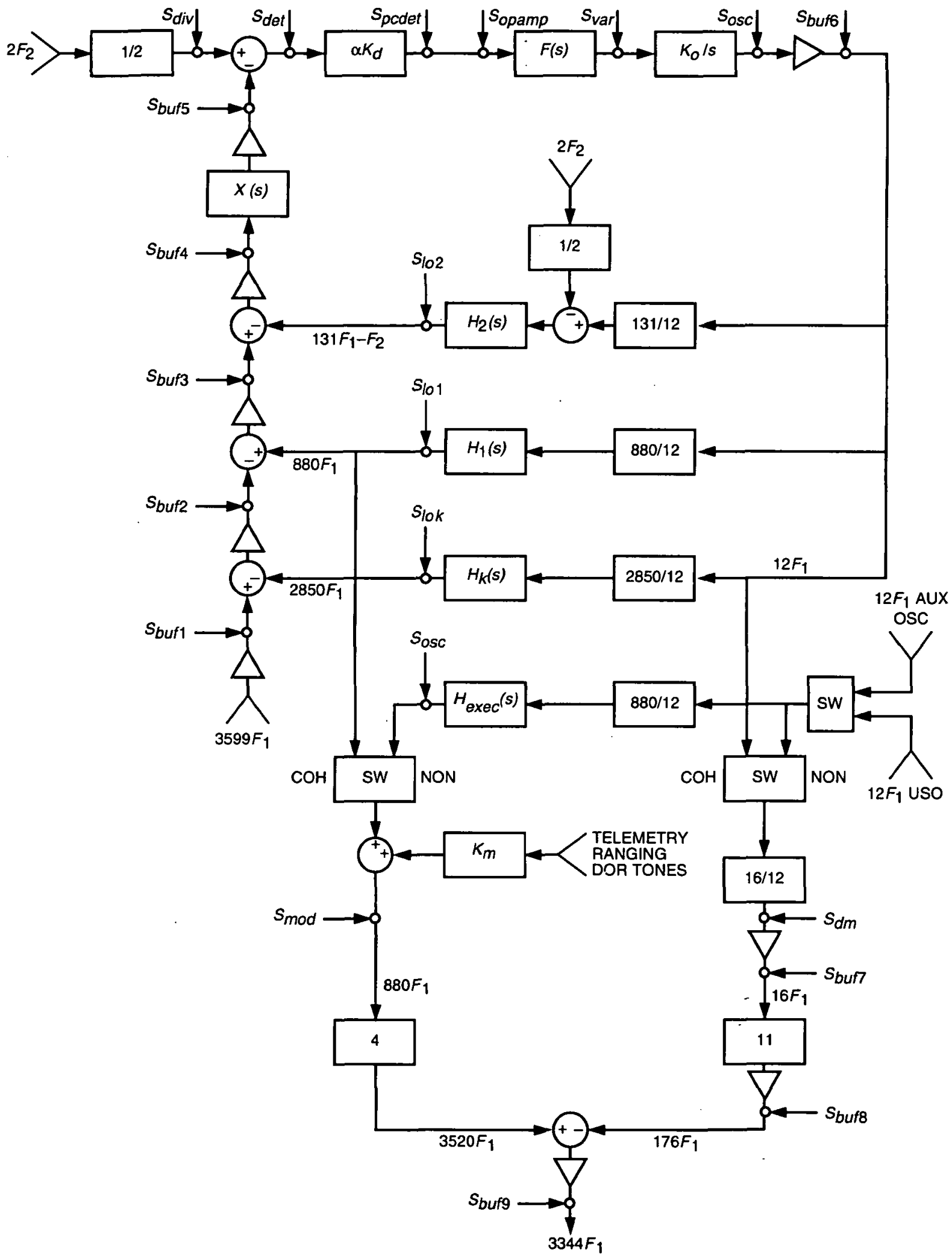

Fig. A-1. Phase noise model for the Ka-band receiver and exciter circuit. 
(3) Power Converter: The phase noise model for the power converter is obtained from empirical data measured on the Tracking Data Relay Satellite Systems (TDRSS) secondgeneration user transponder.

(4) Regulator: The regulator is modeled as a high-pass filter response and a thermal noise floor. The high-pass filter response of the regulator reduces the power converter noise at low frequencies to that of the reference zener diode.

(5) Varactors: The phase noise of varactors is modeled as the regulator output noise plus a thermal noise floor.

(6) Phase Modulator: The phase noise density of the phase modulator is caused by the power converter noise directly modulating the downlink and by the regulator noise driving the varactors.

\section{Downlink Noise Density at Ka-Band}

The downlink phase noise density is shaped by the closed-loop transfer function of the receiver $\left(H_{c}\right)$, the error transfer function of the receiver $\left(H_{e}\right)$, and the transfer function from the input of the crystal filter to the output of the receiver $\left(H_{x}\right)$. These transfer functions are given by

$$
\left.\begin{array}{l}
H_{c}=\frac{H_{o}}{1+H_{o} H_{x t a l} H_{r}} \\
H_{e}=\frac{1}{1+H_{o} H_{x t a l} H_{r}} \\
H_{x}=\frac{H_{o} H_{x t a l}}{1+H_{o} H_{x t a l} H_{r}}
\end{array}\right\}
$$

where

$$
\left.\begin{array}{l}
H_{o}=\frac{\alpha K_{d} K_{o} H_{f l t}}{s} \\
H_{r}=\frac{880}{12} H_{l o 1}-\frac{131}{12} H_{l o 2}+\frac{2850}{12} H_{l o k}
\end{array}\right\}
$$

In these equations, $\alpha$ is the signal suppression factor due to the limiter $[1,5,6], H_{f l t}$ is the receiver-loop filter transfer function, $H_{l o 1}$ is the DST-AEM receiver X-band DRO-loop transfer function, $H_{l o 2}$ is the DST-AEM receiver S-band SRO-loop transfer function, $H_{l o k}$ is the Ka-band DRO-loop transfer function, and $H_{x t a l}$ is the crystal filter transfer function. The phase-noise power spectral density at the output of the $12 F_{1}$ phase-locked VCO is given by

$$
S_{12 f}=S_{1}\left|H_{c}\right|^{2}+S_{2}\left|H_{e}\right|^{2}+S_{3}\left|H_{x}\right|^{2}
$$

where 


$$
\left.\begin{array}{l}
S_{1}=S_{b u f 5}+S_{d e t}+S_{d i v}+\frac{S_{p c d e t}+S_{o p a m p}}{\alpha K_{d}^{2}} \\
S_{2}=S_{o s c}+S_{b u f 6}+S_{v a r}\left(\frac{K_{o}}{f}\right)^{2} \\
S_{3}=S_{b u f 1}+S_{b u f 2}+S_{b u f 3}+S_{b u f 4}+S_{l o 1}+S_{l o 2}+S_{l o k}
\end{array}\right\}
$$

In these equations, $S_{b u f i}, S_{d e t}, S_{p c d e t}, S_{o p a m p}, S_{d i v}, S_{v a r}, S_{l o 1}, S_{l o 2}, S_{l o k}$, and $S_{o s c}$ denote the phase noise contributions of the $i$ th buffer amplifier, the phase detector, the power converter at the phase detector, the operational amplifier, the divide-by-two divider, the varactor dicde, the DST-AEM receiver X-band DRO loop, the DST-AEM receiver S-band SRO loop, the Ka-band DRO loop, and the DST-AEM receiver $12 F_{1} \mathrm{VCO}$, respectively. The remaining symbols, $K_{d}, K_{o}$, and $f$, denote the phase detector sensitivity in $\mathrm{V} / \mathrm{rad}$, the $12 F_{1} \mathrm{VCO}$ sensitivity in $\mathrm{Hz} / \mathrm{V}$, and the frequency in $\mathrm{Hz}$, respectively.

The phase noise density at the receiver PLL VCO output is used in the exciter to estimate the coherent Ka-band downlink phase noise density. For noncoherent mode, the downlink phase noise density is obtained by replacing the PLL VCO phase noise density $\left(S_{12 f}\right)$ by the AUX OSC or USO phase noise density. Thus, the Ka-band downlink phase noise density at $3344 F_{1}$ is given by

$$
S_{3344 f}=4^{2} S_{12 f}\left|\frac{220}{3} H_{880 f}-\frac{11}{3}\right|^{2}+4^{2}\left(S_{880 f}+S_{m o d}\right)+11^{2}\left(S_{b u f 7}+S_{d m}\right)+S_{b u f 8}+S_{b u f 9}
$$

where $H_{880 f}$ and $S_{880 f}$ are, respectively, the transfer function and the phase noise density of the DST-AEM exciter X-band DRO loop for noncoherent operation or the receiver X-band DRO loop for coherent operation; $S_{m o d}$ is the phase noise contribution of the X-band phase modulator; and $S_{d m}$ is the phase noise contribution of the $16 / 12$ divider-multiplier circuit.

\section{Appendix B}

\section{Carrier-Tracking Threshold Calculations}

The equations used to calculate carrier-tracking threshold data are presented in this appendix. The carrier-tracking threshold of a PLL receiver $[1,6,7]$ is defined as the minimum uplink signal required to maintain lock at any given offset from best-lock frequency. It is a measure of an important limitation on spacecraft receiver performance. At the best-lock frequency, the carrier-tracking threshold signal level is determined from the following equation:

$$
\frac{S L}{k T F\left(2 B_{L O}\right)}=1
$$

where $S$ is the receiver input-signal power level, $k$ is Boltzmann's constant, $T$ is the reference system temperature, $F$ is the receiver noise figure at the transponder input, $B_{L 0}$ is the one-sided noise-equivalent receiver carrier-tracking loop bandwidth at threshold, and $L$ is the receiver carrier channel loss. The 
calculated value of the worst-case receiver carrier-tracking threshold is equal to $-149 \mathrm{dBm}$ for a $2 B_{L 0}$ of $72 \mathrm{~Hz}$, channel loss of $1 \mathrm{~dB}$, and a noise figure of $5.4 \mathrm{~dB}$ (including isolator, filter, LNA, and connectors) at $290 \mathrm{~K}$.

Note that the carrier-tracking threshold signal level can also be expressed in terms of the variance of the phase error due to additive channel noise by

$$
\sigma_{\theta}^{2}=\frac{k T F B_{L O}}{S L}=\frac{1}{2}
$$

Therefore, at threshold, the standard deviation of the phase error is $\sigma_{\theta}=0.707$. If the uplink signal frequency is offset from the best-lock frequency by $\Delta f \mathrm{~Hz}$, the phase detector is required to operate with a static phase error of $\Theta_{e}$ rad, given as

$$
\Theta_{e}=\sin ^{-1}\left(\frac{2 \pi \Delta f}{\alpha K_{v}}\right)
$$

where $K_{v}$ is the dc gain of the PLL and $\alpha$ is the receiver limiter suppression factor, given by

$$
\alpha=\frac{1}{\sqrt{1+\frac{4}{\pi} \frac{k T F B_{i f}}{S L}}}
$$

Here $B_{i f}$ is the noise-equivalent predetection bandwidth. This static phase error reduces the threshold phase-error standard deviation to $\sigma_{\theta}=0.707-\Theta_{e}$, such that the carrier-tracking threshold corresponding to the frequency offset of $\Delta f \mathrm{~Hz}$ needs to satisfy

$$
\sigma_{\theta}^{2}=\frac{k T F B_{L O}}{S L}=\left(\frac{1}{\sqrt{2}}-\Theta_{e}\right)^{2}
$$

The one-sided noise-equivalent carrier-tracking loop bandwidth at threshold, $B_{L 0}$, is given by

$$
B_{L O}=\frac{1+\frac{K \tau_{2}^{2}}{\tau_{1}}}{4 \tau_{2} \frac{1+1}{K \tau_{2}}}
$$

where $\tau_{1}$ and $\tau_{2}$ are the loop filter time constants and $K$ is the open loop gain of the PLL, given by

$$
K=\alpha K_{v} \cos \left(\Theta_{e}\right) e^{-0.5\left(0.707-\Theta_{e}\right)^{2}}
$$

Note that the open loop gain of the PLL includes factors for the reduction of the phase detector sensitivity due to the static phase error and the additive channel noise. 